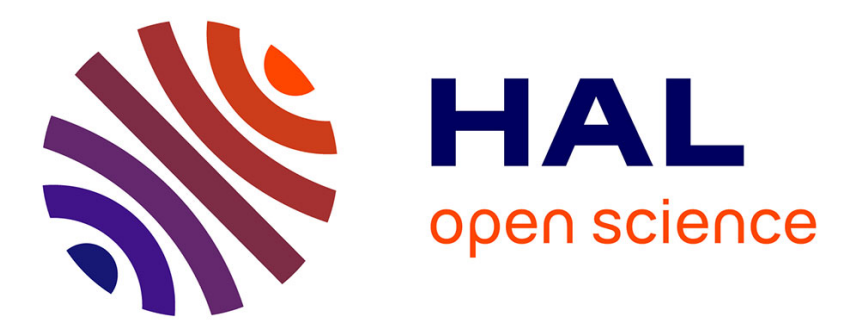

\title{
A neuro-fuzzy self built system for prognostics : a way to ensure good prediction accuracy by balancing complexity and generalization.
}

\author{
Mohamed El-Koujok, Rafael Gouriveau, Noureddine Zerhouni
}

\section{- To cite this version:}

Mohamed El-Koujok, Rafael Gouriveau, Noureddine Zerhouni. A neuro-fuzzy self built system for prognostics : a way to ensure good prediction accuracy by balancing complexity and generalization.. IEEE Prognostics \& System Health Management Conference, PHM'2010., Jan 2010, Macau, China. 8 p. hal-00459285

\author{
HAL Id: hal-00459285 \\ https://hal.science/hal-00459285
}

Submitted on 23 Feb 2010

HAL is a multi-disciplinary open access archive for the deposit and dissemination of scientific research documents, whether they are published or not. The documents may come from teaching and research institutions in France or abroad, or from public or private research centers.
L'archive ouverte pluridisciplinaire HAL, est destinée au dépôt et à la diffusion de documents scientifiques de niveau recherche, publiés ou non, émanant des établissements d'enseignement et de recherche français ou étrangers, des laboratoires publics ou privés. 


\title{
A Neuro-Fuzzy Self Built System For Prognostics: a Way To Ensure Good Prediction Accuracy by Balancing Complexity and Generalization
}

\author{
Mohamed El-Koujok, Rafael Gouriveau, Noureddine Zerhouni \\ FEMTO-ST Institute, UMR CNRS 6174 - UFC / ENSMM / UTBM \\ Automatic Control and Micro-Mechatronic Systems Department \\ 24, rue Alain Savary, 25000 Besançon, France \\ \{mohamed.elkoujok, rgourive, zerhouni\}@ens2m.fr
}

\begin{abstract}
In maintenance field, prognostics is recognized as a key feature as the prediction of the remaining useful life of a system allows avoiding inopportune maintenance spending. However, it can be a non trivial task to develop and implement effective prognostics models including the inherent uncertainty of prognostics. Moreover, there is no systematic way to construct a prognostics tool since the user can make some assumptions: choice of a structure, initialization of parameters... This last problem is addressed in the paper: how to build a prognostics system with no human intervention, neither a priori knowledge? The proposition is based on the use of a neuro-fuzzy predictor whose architecture is partially determined thanks to a statistical approach based on the Akaike information criterion. It consists in using a cost function in the learning phase in order to automatically generate an accurate prediction system that reaches a compromise between complexity and generalization capability. The proposition is illustrated and discussed.
\end{abstract}

\section{INTRODUCTION}

The growth of reliability, availability or safety of a system is a determining factor in regard with the effectiveness of industrial maintenance. Thus, the implementation of provisional strategies is a good way to improve the availability of processes, to ensure the smallest variations of products qualities or the direct costs falling [1]. Furthermore, durable development involves the integration of economical strategies beside social and environmental ones for the optimization of processes. This major provocation of triple performance outlined a new and interesting research area: concepts like corrective or preventive maintenance were progressively completed by predictive and proactive maintenance [2] and prognostics is now recognized as a key feature in maintenance strategies as the estimation of the provisional reliability of equipment as well as its remaining useful life allows avoiding inopportune spending.

A central problem can be pointed out: the accuracy of a prognostics system is related to its ability to predict the degradation of equipment: starting from a "current situation", a prognostics tool must be able to forecast the "future possible situations". From the research point of view, many developments exist to support these prognostics or forecasting activities $[3,4,5,6]$. However, choosing an efficient technique depends on classical constraints that limit the applicability of the tools: available data-knowledge-experiences, complexity and dynamic of systems, available monitoring devices, implementation requirements (precision, computation time...).

Following that and according to literature, neuro-fuzzy (NF) systems appear to be very promising prognostic tools: NFs learn from examples and attempt to capture the subtle relationships among data. NFs are computationally effective techniques and are thereby well suited for practical problems, where it is easier to gather data than to formalize the behavior of the system being studied. Actual developments confirm the interest of using NFs in forecasting applications [7, 8]. (Note that the purpose of the authors is not to present NFs systems as the single tools for prognostics, but as adequate to face with the implementation restriction and requirements.)

Nevertheless and in spite of the capabilities of NFs systems, building a NFs model for prediction is not a trivial task: various fuzzy structures can be used, the nature and quantity of inputs, as well as the form of the membership's functions have to be chosen, different learning algorithms exist, a random initialization (or expert made) must be done... Moreover, these choices are critical as they directly have an impact on the accuracy of the predictions of the system. This is the problem addressed in this paper: how to build a prognostics system with no human intervention, neither a priori knowledge?

The proposition is based on the use of an evolving NFs system that starts from scratch, i.e., for which any assumption on its structure is necessary. A statistical approach based on the Akaike criterion (AIC) is used in order to automatically generate an accurate prediction system that reaches a compromise between complexity and generalization capability.

The paper is organized in four main parts. First, the concept of prognostics is clarified in order to provide the lector with the limit of this work. As the efficiency of a prognostics system is highly dependent on its ability to perform good predictions, the relationship between prognostics and prediction is also explained and the use of first order Takagi-Sugeno neuro-fuzzy systems in prognostics applications is justified. Following that, the next section is dedicated to the proposition of a self built NFs system for prognostics. For that purpose, the ways of building such models are discussed as well as the learning aspects that influence the prediction performances and an evolving neuro fuzzy system is pointed out. The method to balance complexity and generalization capability is then presented and the whole is finally illustrated in the last part: various experiments on industrial predictions benchmarks are used in order to discuss the proposition and to compare it with the classical Auto Regressive eXogenous approach (ARX). 


\section{TOWARDS NF-SYSTEMS FOR PROGNOSTICS}

\section{The concept of prognostics}

Prognostics is traditionally related to fracture mechanics and fatigue. It started to be brought up by the modal analysis community as an intersting field [9]. In this "meaning", prognostics is called the prediction of a system's lifetime. Prognostics can also be defined as a probability measure: the chance that a machine operates without a fault or failure up to some future time [10]. This "probabilistic prognostics value" is all the more an interesting indication as the fault or failure can have catastrophic consequences (e.g. nuclear power plant) and maintenance manager need to know if inspection intervals are appropriate. Some authors introduce prognostics as a process that allows the a priori reliability modeling and thereby enables to estimate the remaining time to underpass a limit fixed by the practitioner or by past experiences $[11,12]$.

Finally, although there are some divergences in literature, prognostics can be defined as proposed by the International Organization for Standardization: "prognostics is the estimation of time to failure and risk for one or more existing and future failure modes" [13]. In this acceptation, prognostics is also called the "prediction of a system's lifetime" as it is a process whose objective is to predict the remaining useful life (RUL) before a failure occurs given the current machine condition and past operation profile [5].

\section{Prognostics and prediction}

All definitions proposed here before assimilate prognostics to a "prediction process": a future situation must be caught. It obviously supposes that the current situation can be grasped (practically, it's the synthesis of a detection process and of measured data of the system). Following that and according to previous works, two salient aspects of prognostics appear [14]:

1. prognostics is mostly assimilated to a prediction process (a future situation must be caught),

2. prognostics is based on the failure notion, which implies that it is associated with a limit of acceptability (the predicted situation must be assessed with regard to a referential).

Thus, prognostics could be split into 2 sub-activities: a first one to predict the evolution of a situation at a given time, and a second one to assess this predicted situation with regards to an evaluation referential. Let's resume (figure 1):

- identification: a situation is captured by the detection process and additional current measures,

- prediction: the situation is forecasted in time,

- assessment: a situation is evaluated by the use of performance criteria,

- prognostics: a predicted situation is assessed.

In addition, a central problem appears: the accuracy of a prognostics system is related to its ability to approximate and predict the degradation of an equipment; the prediction phase is a critical one. Developments of this paper emphasizes on this aspect of prognostics. In pratice, the prediction step must be supported by operational tools which choice is important.

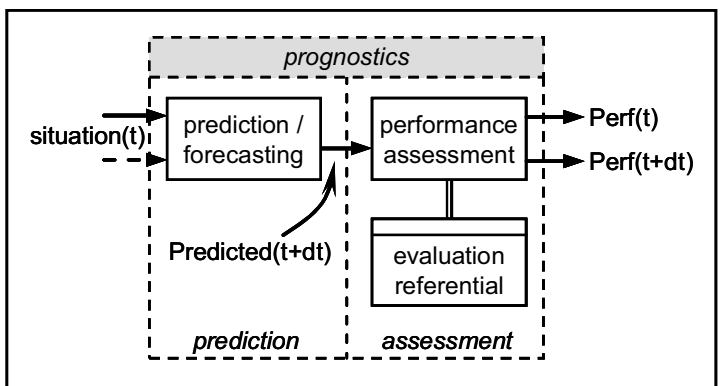

Fig. 1 Prognostics as a prediction and assessment process [14]

\section{First order TS systems for prediction / forecasting}

The aim of this part is not to dress an exhaustive overview of prediction approaches but to explain the orientations of works that have been taken. For more details on prognostics approaches, see $[6,15]$.

Real systems are complex and their behaviour is often non linear, non stationary. These considerations make harder a modelling step, even impossible. Yet, a prediction tool must deal with it. Moreover, monitoring systems have evolved and it is now quite easy to online gather data. According to all this, data-driven approaches have been increasingly applied to prediction problems in general and to machine prognostics in particular, and works emphasize on the interest of using hybrid systems for prediction purpose. More precisely, first order Takagi-Sugeno (TS) fuzzy models have shown improved performances over conventional approaches $[7,8]$, and they appear to be adequate to perform the degradation modeling step of prognostics [11].

\section{First order Takagi-Sugeno systems: principle}

A first order TS model can be seen as a multi-model structure consisting of linear models that are not necessarily independent [16]. It is based on the fuzzy decomposition of the input space. For each part of the state space, a fuzzy rule can be constructed to make a linear approximation of the input. The global output is a combination of the whole rules.

A TS model is composed of 5 layers. Consider figure 2. This model has two inputs variables. Two membership functions (antecedent fuzzy sets) are assigned to each one of them. The TS model is finally composed of two fuzzy rules. (That can be generalized to the case of $\mathrm{n}$ inputs and $\mathrm{N}$ rules). The rules perform a linear approximation of inputs:

$R_{i}$ : if $x_{1}$ is $A_{i}^{l}$ and ... and $x_{n}$ is $A_{i}^{n}$

THEN $y_{i}=a_{i 0}+a_{i 1} \cdot x_{1}+\ldots+a_{i n} \cdot x_{n}$

where $R_{i}$ is the $i^{\text {th }}$ fuzzy rule, $N$ is the number of rules, $X_{t}=\left[x_{1}, x_{2}, \ldots, x_{n}\right]^{T}$ is the input vector, $A_{i}^{j}$ denotes the antecedent fuzzy sets, $j=[1, n], y_{i}$ is the output of the $i^{\text {th }}$ linear subsystem, and $a_{i q}$ are its parameters, $q=[1, n]$.

In layer 1, let assume Gaussian antecedent fuzzy sets to define the regions of fuzzy rules in which the local linear submodels are valid:

$\mu_{j}^{i}=\exp \left(-4\left\|x-x^{i *}\right\|_{j} /\left(\sigma_{j}^{i}\right)^{2}\right)$ 


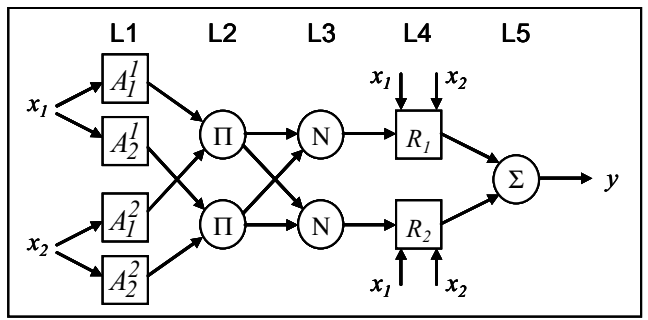

Fig. 2 A First-order TS model

where $\sigma_{i}^{j}$ is the spread of the membership function, and $x^{i *}$ is the focal point (center) of the $i^{\text {th }}$ rule antecedent. The firing level $\left(\tau_{i}\right)$ and the normalized firing level $\left(\lambda_{i}\right)$ of each rule are obtained as follows (layers 2 and 3 ):

$\tau_{i}=\mu_{i 1}\left(x_{1}\right) \times \ldots \times \mu_{i n}\left(x_{n}\right), \lambda_{i}=\tau_{i} / \sum_{j=1}^{N} \tau_{j}$

The model output is the weighted averaging of individual rules' contributions (layers 4 and 5):

$y=\sum_{i=1}^{N} \lambda_{i} y_{i}=\sum_{i=1}^{N} \lambda_{i} x_{e}^{T} \pi_{i}$

where $\pi_{i}=\left[\begin{array}{llll}a_{i 0}, & a_{i 1}, \ldots, a_{i n}\end{array}\right]$ is the vector parameter of the $i^{t h}$ sub-model, and $x_{e}=\left[\begin{array}{ll}1 & X^{T}\end{array}\right]^{T}$ is the expanded data vector.

A TS model has two types of parameters. The non-linear parameters are those of the membership functions (the center and the spread deviation for a Gaussian membership). These kinds of parameter are referred to as premise or antecedent parameters. The second types of parameters are the linear ones that form the consequent part of each rule ( $a_{i q}$ in eq. 1$)$.

\section{A SELF BUILT NF SYSTEM FOR PREDICTION}

\section{Fitting a neuro-fuzzy systems: critical steps}

Assuming that a TS model can approximate an input-output function, in practice, this kind of model must be tuned to fit to the studied problem (prediction in our case). This implies at least four complementary tasks to be performed.

1. Choice of input signals. This step aims at determining which variables are relevant to model the desired input-output relation, i.e., to identify the exogenous variables of the phenomenon. It can be expert made or result from processing techniques (feature extraction and selection). This problem is not addressed in this paper, but for subsequent explanations, let note $X=\left[x_{1}, x_{2}, \ldots, x_{n}\right]$ the vector of exogenous variables that enables to estimate $y_{d}$, the endogenous variable.

2. Inputs definition. Time series predictions are usually made by considering some past values of each variable. In order to make a prediction, one has to select the set of regressors for modeling $\left(\varphi_{s}\right)$, within the global set of potential regressors $(\varphi)$ of both the exogenous and endogenous variables. Formally, and assuming $h$ the horizon of prediction, $\theta$ the vector parameter of the model, at any time $t$, prediction for time $t+h$ can be expressed as in equation 5 .

3. Design of the structure. In a few worlds, one has to choose the number and type of membership functions for each input (layer 1) and the number of rules which depends on the numbers of connections in between layers 2 and 3. The design of a TS model is thereby quite flexible. Nevertheless, choices made at this step directly influence the accuracy performance of the model as well as its complexity (as more membership functions and rules are chosen, as more parameters have to be tuned). Note that thanks to its generalization capabilities, Gaussian membership's functions are usually used.

4. Fitting the parameters. The parameters of a TS model must be tuned to fit to the studied problem. This is the aim of the learning procedure and different approaches can be used. In all cases, the consequent parameters of the system (the linear ones) are tuned by using a least squares approach.

$$
\hat{y}(t+h)=f\left(\varphi_{s}, \theta\right), \quad \varphi_{s} \in \varphi=\left\{\begin{array}{c}
y_{d}(t-1) \ldots y_{d}\left(t-q_{y}\right) \\
x_{1}(t-1) \ldots x_{1}\left(t-p_{x_{I}}\right) \\
\ldots \\
x_{n}(t-1) \ldots x_{n}\left(t-p_{x_{n}}\right)
\end{array}\right.
$$

\section{Reducing human influence thanks to evolving algorithms}

Whereas the first of these four steps is not studied in this paper, the second one is discussed in section IV. Followings paragraphs present the ways of identifying TS model and enables to discuss the above points 2 and 3 .

The simplest method to construct a TS fuzzy system is the "mosaic scheme": the user defines the architecture of the model and the antecedent's parameters values [17]. This approach must be leaved aside to reduce the influence of practitioner. Gradient descent algorithms have been adapted to the TS structure in order to calculate the antecedent parameters by the standard back-propagation procedure (see for example the ANFIS system proposed by [18]). In the same way, genetic algorithms can be used to compute the fitting of antecedent parameters. These approaches require the user to choose the number of membership's functions, and to initialize various algorithms parameters. To face with it, clustering approaches (and some genetic algorithms) have been adapted to neurofuzzy systems, the basic idea being to use a learning data set to automatically generate the adequate structure of the TS model (number of membership functions, and of rules). However, in practical applications, the learning process is effective only if sufficient data are available and, when trained, such a TS model is fixed. Thereby, if the behavior of input and/or output changes significantly with regards to the learning phase (like in a degradation process), predictions can suffer from the lack of representative learning data. In order to continuously integrate the dynamic of signals, evolving algorithms have finally been developed [16, 19]. These algorithms are based on clustering methods and therefore, do not require the user to define the structure of the TS model. In opposition to all previous approaches, they do not need a complete learning data set to start the identification process of the TS model (start from scratch): they have a flexible structure that evolves with the data gathered from the system. Data are collected continuously which enables to form new rules or to modify an existing one.

This kind of self constructing evolving predictors are thereby very interesting for prognostics applications where it is very difficult, even impossible, to formalize the behavior of the system. A particular evolving TS model is that one proposed by $[16,20]$ : the evolving extended TS system (exTS). 


\section{Theoretical exTS backgrounds}

The learning procedure of exTS is composed of two phases: (1) an unsupervised data clustering technique is used to adjust the antecedent parameters, (2) the supervised recursive least squares learning method updates consequent parameters. Both algorithms can not be fully detailed in this paper.

The exTS clustering phase processes on the global inputoutput data space: $z=\left[x^{T} ; y^{T}\right]^{T}, z \in R^{n+m}$, where $n+m$ defines the dimensionality of the input/output data space. Each one of the sub-model of exTS operates in a sub-area of $z$. This clustering algorithm is based on the calculus of a potential which is the capability of a data to form a cluster (antecedent of a rule). The procedure starts from scratch and, as more data are available, the model evolves by replacement or upgrade of rules. This enables the adjustment of the antecedent parameters.

The exTS model is used for online prediction: at step $k$, equation 4 can be expressed as follows:

$\hat{y}_{k+1}=\sum_{i=1}^{N} \lambda_{i} y_{i}=\sum_{i=1}^{N} \lambda_{i} x_{e}^{T} \pi_{i}=\psi_{k}^{T} \hat{\theta}_{k}$

$\psi_{k}^{T}=\left[\lambda_{1} x_{e}^{T}, \lambda_{2} x_{e}^{T}, \ldots, \lambda_{n} x_{n}^{T}\right]_{k}^{T}$ is a vector of the inputs, weighted by normalized firing $(\lambda)$ of the rules, and $\hat{\theta}_{k}=\left[\hat{\pi}_{1}^{T}, \hat{\pi}_{2}^{T}, \ldots, \hat{\pi}_{N}^{T}\right]_{k}^{T}$ are parameters of the sub-models.

An estimation of the parameters based on $k$ data samples is obtained by applyong the following RLS procedure:

$\hat{\theta}_{k}=\hat{\theta}_{k-1}+C_{k} \psi_{k}\left(y_{k+1}-\psi_{k}^{T} \hat{\theta}_{k-1}\right) ; k=2,3, \ldots$

$C_{k}=C_{k-1}-C_{k-1} \psi_{k} \psi_{k}^{T} C_{k-1} / 1+\psi_{k}^{T} C_{k-1} \psi_{k}$

with initial conditions $\theta_{1}=\left[\pi_{1}^{T}, \pi_{2}^{T}, \ldots, \pi_{N}^{T}\right]_{k}^{T}=0, C_{1}=\Omega I$, where $\Omega$ is a large positive number, $\Omega I$ a $R(n+1) \times R(n+1)$ covariance matrix.

\section{Complexity of an exTS system}

The architecture of an exTS neuro-fuzzy system is singular in that the number of rules is equal to the number of membership's functions by input. Thereby and assuming that exTS is a firstorder TS model, the number of linear parameters can be simply determined by the relation $n b_{l}=N \times(n+1)$, where, $N$ is the number of rules and $n$, the number of inputs. As being Gaussian, each membership has 2 parameters to be tuned and the total amount of non-linear parameters is expressed by $n b_{n l}=2 \times n \times N$.

The total number of parameters of an exTS can be interpreted as an indicator of the complexity of the model:

$n b_{\text {exTS }}=n b_{l}+n b_{n l}=N(3 \times n+1)$

\section{BALANCING COMPLEXITY AND GENERALIZATION CAPABILITY}

\section{Problem statement}

According to previous work $[21,22]$, exTS is an effective forecasting tool: it can perform accurate predictions while minimizing the user's assumptions. Nevertheless, whereas the number of rules is automatically computed in the clustering phase of the exTS learning procedure, the number of inputs must be set by the practitioner (which is the right set of regressors? - see the second critical step defined in previous page). According to equation 8 , this implies a more or less complex fuzzy structure and the prediction performance follows from it. Thereby, the purpose of this section is to propose a way to automatically generate an accurate exTS prediction system that reaches a compromise between complexity and generalization capability. The approach is based on the Akaike Information Criterion (AIC).

\section{Akaike Information Criterion}

Akaike Information Criterion (AIC) has been introduced in order to provide a mathematical formulation of the principle of parsimony in the field of model construction [23]. This criterion enables to judge from the quality of fit of an estimator and can be used with prediction models. It is defined as follows: $A I C=S \times \log (L)+2 \times p$

where, $S$ is the number of data samples used when fitting the model, $p$, the number of independently adjusted parameters within the model, $L$ a likelihood function of the model, i.e., a loss function that traduces the accuracy of past estimations:

$L=\frac{1}{S} \sum_{i=1}^{S}\left(y_{d}-\hat{y}\right)^{2}$

According to equations 9 and 10, AIC is composed of two parts. The first one $(S \cdot \log (L))$ enables to weight the importance of the accuracy of the estimator (/ predictor), whereas the second one $(2 \times p)$ aims at weighting the complexity of the model:

- AIC is as low, as the accuracy is good (logarithmically),

- AIC is as high, as the number of parameters is high (linearly).

Various identification / prediction models can be ranked according to their AIC, the more suitable been that of the lowest ones (suitable in the sense of "compromise in between accuracy and complexity"). Consider figure 3 for an illustration.

Note that the AIC criterion can be implemented without the aid of subjective judgment.

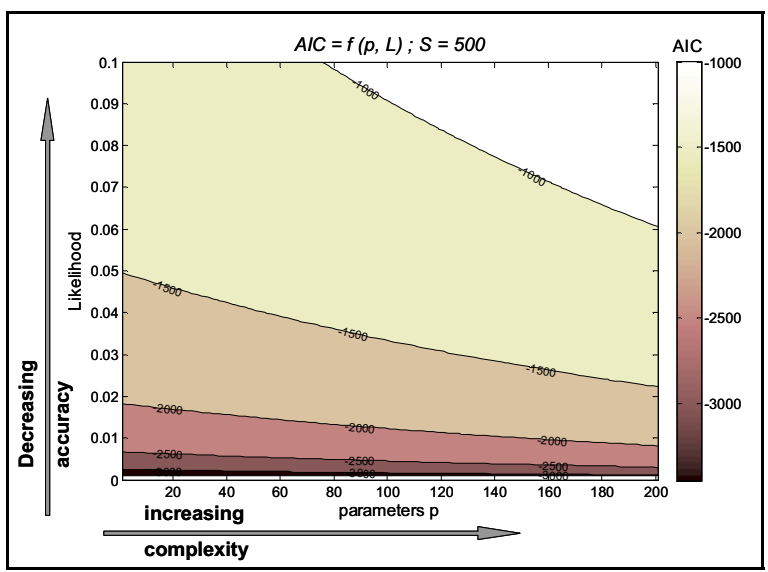

Fig. 3 Evolution of AIC

Balancing complexity and accuracy of an exTS predictor As stated in equation 5, in order to build a prediction model, one has to define the set of regressors of both the exogenous and endogenous variables. 
Let note $O=\left\{p_{x 1}, p_{x 2}, \ldots, q_{y}\right\}$ the order of the model containing the number of past values used for prediction for each input. Depending on this choice, many forecasting systems can be constructed. In addition, and thanks to equation 8 , the AIC of all these potential models can be easily computed during the learning phase. Following that, one is able to determine which model presents the lowest AIC and is thereby providing a compromise between complexity and accuracy. This procedure is depicted in figure 4.

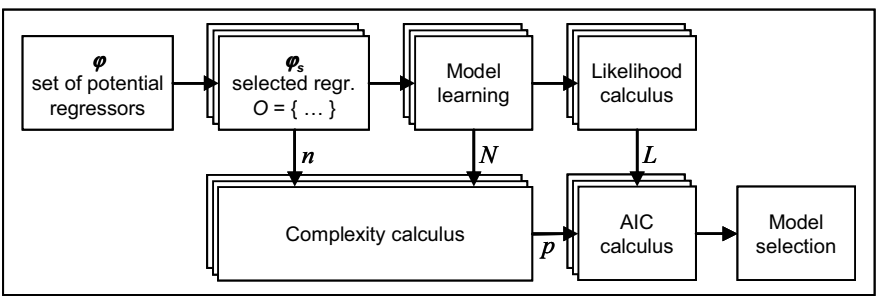

Fig. 4 Procedure to build a "suitable" exTS

\section{EXPERIMENTS AND DISCUSSION}

\section{Object}

The aim of previous sections was to point out a suitable prediction technique for prognostics purpose, for which any assumption or intervention of the practitioner is necessary, i.e., to propose a way to go through self built systems. That can be reached thanks to two aspects that are illustrated in this section:

- the evolving exTS model is an accurate prediction tool that outperforms classical approaches,

- the procedure of input selections derived from the AIC criterion enables to automatically generate a fuzzy architecture that balance accuracy and complexity.

The Auto Regressive eXogenous approach (ARX) has been used for comparisons: this model is well known and extensively employed in modeling and prediction of times series. Given a set of inputs $x_{i}$, an ARX prediction can be expressed as:

$$
A(w) \cdot \hat{y}(t)=\sum_{i=1}^{n} B_{i}(w) \cdot x_{i}(t)
$$

where, $A(w)=1+\alpha_{1} w^{-1}+\cdots+\alpha_{q y} w^{-q_{y}}$

$$
\begin{aligned}
& B_{i}(w)=\beta_{1}^{i} w^{-1}+\cdots+\beta_{p_{x_{i}}}^{i} w^{-p_{x_{i}}} \\
& w^{-\tau} \cdot x(t)=x(t-\tau)
\end{aligned}
$$

As for the exTS model, let note $O=\left\{p_{x 1}, p_{x 2}, \ldots, q_{y}\right\}$ the order of an ARX model, with total amount of parameters:

$$
n b_{A R X}=q_{y}+p_{x_{1}}+\cdots+p_{x_{n}}
$$

Two real experimental data sets have been used for tests. In both cases, the aim of predictions is to approximate a physical phenomenon by learning data gathered from the system. That can be assimilated to the prediction step of the prognostics process.

\section{First data sets: industrial dryer}

The first data set is issued from an industrial dryer. It has been contributed by Jan Maciejowski from Cambridge
University [24]. Three exogenous variables $\left(x_{1}, x_{2}, x_{3}\right)$ are linked to one endogenous variable $(y)$ :

- $x_{1}$ : fuel flow rate,

- $x_{2}$ : hot gas exhaust fan speed,

- $\quad x_{3}$ : rate of flow of raw material,

- $\quad y$ : dry bulb temperature.

This data set has been used to evaluate the ability of an exTS system to predict a non-linear function. This has been made with comparison with the ARX model. For both models, the procedure of input selection based on AIC criterion has been applied in order to identify the a priori more suitable structure, i.e., that one that balances complexity and accuracy (let name them the exTS comp and $A R X_{\text {comp }}$ models). According to equations 8 and 12, the complexity of the models can be easily calculated. The prediction performance was assessed by using the root mean square error criterion (RMSE) which is the most popular prediction error measure and the coefficient of determination $(R 2)$ which is a measure of how well future outcomes are likely to be predicted by the model:

The data set contains 876 samples. The learning phase was stopped after 500 data samples, and the remaining samples served to test the models at one step ahead. Results are shown in table 1 and figure 5.

\begin{tabular}{|r|cc|}
\hline & $\boldsymbol{A R} \boldsymbol{X}_{\text {comp }}$ & exTS $\boldsymbol{T}_{\text {comp }}$ \\
\hline$O=\left\{p_{x 1}, p_{x 2}, p_{x 3}, q_{y}\right\}$ & $\{9,1,6,6\}$ & $\{1,1,2,3\}$ \\
nb parameters $p$ & 22 & 167 \\
AIC & 1350,7 & $-1146,8$ \\
RMSE test & 4,6097 & 0,7748 \\
R2 test & $-4,4846$ & 0,8451 \\
\hline
\end{tabular}

Table 1. Industrial dryer - simulation results

According to accuracy performance indicators and as shown in figure 5, there is no doubt that the ARX model is not able to catch the behaviour of the dry bulb temperature $(R 2=-4,4846$ !), whereas the exTS model presents good results, even if partially defined thanks to the AIC criterion approach.

Considering our final applicative objective (prognostics of failures), to be capable to carry out predictions on such a signal is of good omen: real systems are complex and have generally a nonstationary and non-linear behaviour, what makes difficult a modelling phase. Working with this data series constitutes a first step to the specification of a prognostics system able to take into account the dynamic of real systems without prior knowledge and human intervention.

\section{Second data sets: air temperature in a mechanical system}

The second data set is issued from an hair dryer. It has been contributed by W. Favoreel from the Kuleuven Unversity [25].

The air temperature of the dryer is linked to voltage of the heating device. This data have inside one exogenous variable $\left(x_{1}\right)$ and one endogenous variable $(y)$ :

- $x_{1}$ : voltage of the heating device,

- $y$ : air temperature. 


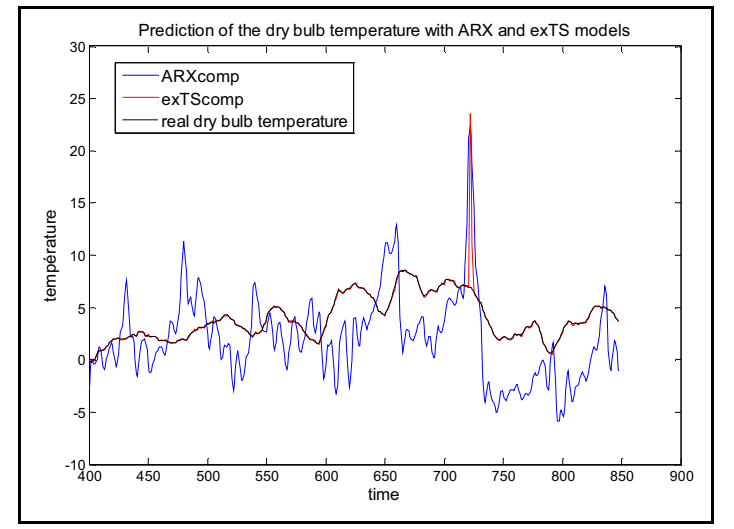

Fig. 5 Industrial dryer - predictions at one step ahead

This data set has been used to evaluate the ability of the input selection procedure based on AIC to reach a compromise between generalization and complexity. Similarly to the first data set, the procedure has been applied to both models ARX and exTS and leaded to two a priori more suitable structures $A R X_{\text {comp }}$ and exTS comp. In order to extract more solid conclusions from the tests and expand the comparison to a same model (and not from one to another), the most accurate models have also been searched by scanning all the set of possible inputs (thanks to the set of potential regressors $\varphi$ ). Let name them the $A R X_{a c c u r}$ and exTS $S_{a c c u r}$ models.

The data set contains 1000 samples. The learning phase was stopped after 500 data samples, and the remaining samples served to test the models at one step ahead. Results are shown in table 2 and figure 6 .

\begin{tabular}{|rcc|}
\hline & $\boldsymbol{A R}_{\boldsymbol{c o m p}}$ & $\boldsymbol{e x T}_{\text {comp }}$ \\
\hline$O=\left\{p_{x l}, q_{y}\right\}$ & $\{18,2\}$ & $\{1,3\}$ \\
nb parameters $p$ & 20 & 117 \\
AIC & $-6,5405$ & -1775 \\
RMSE test & 0,1066 & 0,0962 \\
$R 2$ test & 0,9820 & 0,9854 \\
\hline & $\boldsymbol{A R}_{\boldsymbol{a c c u r}}$ & $\boldsymbol{e x} \boldsymbol{T} \boldsymbol{S}_{\text {accur }}$ \\
\hline O $=\left\{p_{x l}, q_{y}\right\}$ & $\{85,1\}$ & $\{4,3\}$ \\
nb parameters $p$ & 86 & 242 \\
AIC & $-6,3487$ & -1116 \\
RMSE test & 0,0819 & 0,0402 \\
$R 2$ test & 0,9894 & 0,9974 \\
\hline
\end{tabular}

Table 2. Air temperature - simulation results

Results enable to notice that, whatever the model of prediction is, the accuracy grows as the complexity increases: the more the RMSE is near from zero (or $R 2$ near from 1 ), the more parameters the model has. This seems to mean that accuracy and complexity are actually correlated and that there is no way to maximize the satisfaction on both criteria.

Following that, the interest of using the automatic input selection based on AIC can be examined. Whereas the most accurate prediction model for both systems $\left(A R X_{\text {accur }}\right.$ and exTS $\left.S_{\text {accur }}\right)$ has much more parameters than this one obtained by the input selection procedure $\left(A R X_{\text {comp }}\right.$ and exTS $\left.S_{\text {comp }}\right)$, the accuracy of prediction are quite the same. As for an example, consider figure 7 that shows the probability density function (pdf) of the error of prediction for exTS models. Both pdf curves are much closed together. In other worlds, the proposed approach really enables to balance complexity and generalization capability and directly choose an adequate prediction structure.

Remind that it can be very difficult to define a prediction structure for prognostics purpose. The proposed approach, combined with evolving capability of the exTS system provides practitioners with a simple methodology to directly go through a suitable prediction system (self built).

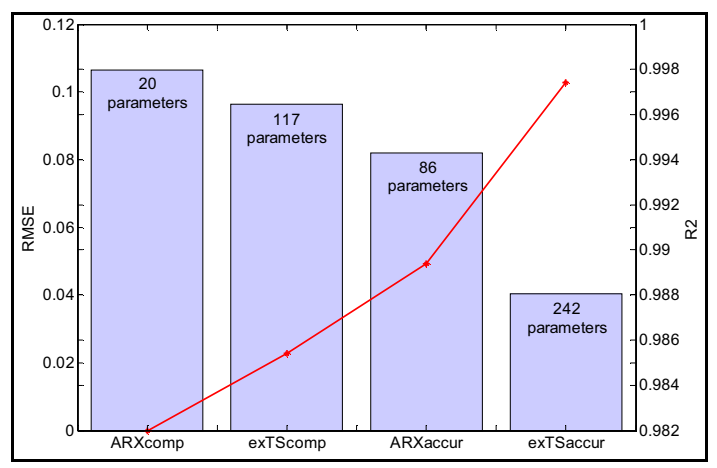

Fig. 6 Air temperature - input selection results

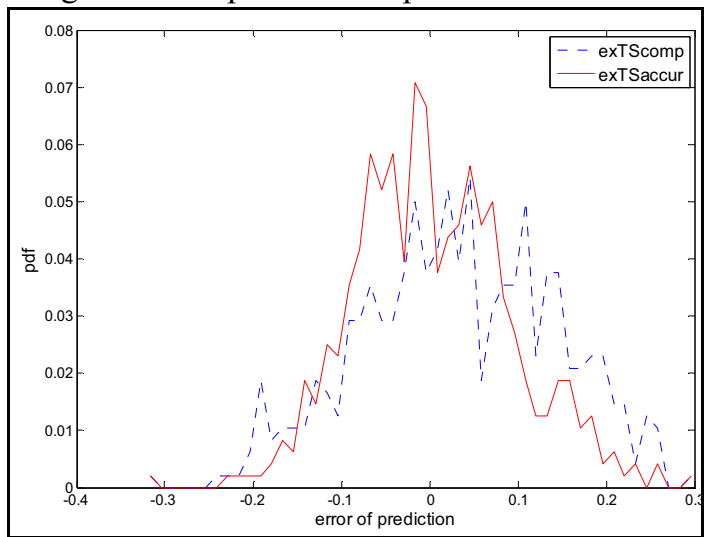

Fig. 7 Pdf of the error of prediction: exTS $S_{\text {comp }}$ and exTS

\section{Conclusions}

The purpose of the work reported in this paper is to point out an accurate prediction technique to perform the approximation and prediction of the degradation of an equipment. Indeed, in one hand, choosing an efficient technique for prognostics depends on constraints that limit the applicability of the tools, and in the other hand, there is no systematic way to construct a prognostics tool since the user can make some assumptions: choice of a structure, initialization of parameters... This is the problem addressed in the paper: how to build a prognostics system with no human intervention, neither a priori knowledge?

According to real implementation restrictions, neurofuzzy systems appear to be well suited for practical problems where it is easier to gather data (online) than to formalize the behavior 
of the system being studied. More precisely, the paper points out the accuracy of the exTS model in prediction. The exTS model has a high level of adaptation to the environment and to the changing data. It is thereby an efficient tool for complex modeling and prediction. Moreover, any assumption on the structure of exTS is necessary, which is an interesting characteristic for practical problems in industry.

Nevertheless, practitioners have to choose the inputs of a NF predictor. In order to face with it, an approach based on the use of the AIC criterion is proposed. It consists in building a cost function that takes into account simultaneously the accuracy of predictions and the complexity of the model. Various inputs can easily be tested in a computational procedure in order to automatically generate an accurate prediction system that reaches a compromise between complexity and generalization capability.

Finally, the whole paper aims at proposing a self-built system for prognostics. Developments are at present extended in order to test the proposition on real degradation data. In addition, other criterions are studied in order to directly integrate the underlying idea of the procedure into the learning algorithms.

\section{References}

1. Leger, JB. and Morel, G. "Integration of maintenance in the enterprise: towards an enterprise modeling based framework compliant with proactive maintenance strategy", Prod. Planning and Control, Vol. 12:2 (2001), pp. 176-187.

2. Muller, A., Suhner, M. and Iung, B. "Formalisation of a new prognosis model for supporting proactive maintenance implementation on industrial system", Reliab. Engineering and System Safety, Vol. 93 (2008), pp. 234-253.

3. Byington, C., Roemer, M., Kacprzynski, G. Galie, T. "Prognostic enhancements to diagnostic systems for improved condition-based maintenance". Proceedings of IEEE Int. Aerospace Conference, Big Sky, USA, 2002.

4. De Gooijer, JG. and Hyndman, RJ. "25 years of time series forecasting", International Journal of Forecasting, Vol. 22 (2006), pp. 443-473.

5. Jardine, AKS., Lin, D. and Banjevic, D. "A review on machinery diagnostics and prognostics implementing condition-based maintenance", Mechanical Systems and Signal Processing, Vol. 20 (2006), pp. 1483-1510.

6. Vachtsevanos, G. et al., Intelligent Fault Diagnostic and Prognosis for Engineering Systems, Wiley \& Sons, (2006).

7. Wang, WQ., Golnaraghi, MF. and Ismail, F. "Prognosis of machine health condition using neuro-fuzzy systems", Mech. Syst. and Sign. Proc., Vol. 18 (2004), pp. 813-831.

8. Yam, RCM., Tse, PW., Li, L. and Tu, P. "Intelligent predictive decision support system for condition-based maintenance, International Journal of Advanced Manufacturing Technology, Vol. 17 (2001), pp. 383-391.

9. Farrar, CR., Hemez, F., Park, G., Robertson, AN., Sohn, H. and Williams, TO. "A Coupled Approach to Developing
Damage Prognosis Solutions". Proceedings International Conf. Damage Assessment of Structures, 2003.

10. Lin, D. and Makis, V. "Recursive filters for a partially observable system subject to random failure", Advances in Applied Probability, Vol. 35 (2003), pp. 207-227.

11. El-Koujok, M., Gouriveau, R. and Zerhouni N. "Error estimation of a neuro-fuzzy predictor for prognostic purpose". Proc. 7th Ifac Int. Sym. SAFE PROCESS, 2009.

12. Chinnam, R. and Pundarikaksha, B. "A neurofuzzy approach for estimating mean residual life in condtitionbased maintenace systems". Int. J. Materials and Product Technology, Vol. 20 (2004), pp. 166-179.

13. ISO, 13381-1. Condition monitoring and diagnostics of machines, prognostics - Part1: General guidelines. International Standard, ISO (2004).

14. Dragomir, O., Gouriveau, R., Zerhouni, N. and Dragomir, F. "Framework for a distributed and hybrid prognostics system". Proc. 4th Ifac Conf MCPL, 2007.

15. Lebold, M. and Thurston M. "Open standards for condition-based maintenance and prognostics systems". Proc 5th Annual Maintenance and Reliability Conf, 2001.

16. Angelov, P. and Filev, D. "An approach to online identification of takagi-sugeno fuzzy models". IEEE Trans. Syst. Man Cybern. - Part B: Cybernetics", Vol. 34 (2004), pp. 484-498.

17. Espinosa, J. et al., Fuzzy Logic, Identification and Predictive Control, Advances in Industrial Control, Springer-Verlag, (2004).

18. Jang, J. and Sun, C. "Neuro-fuzzy modeling and control", IEEE Proceedings, Vol. 83 (1995), pp. 378-406.

19. Kasabov, N. and Song. Q. "Denfis: Dynamic evolving neural-fuzzy inference system and its application for timeseries prediction". IEEE Transaction on Fuzzy Systems, Vol. 10:2 (2002), pp. 144-154.

20. Angelov, P. and Zhou, X. "Evolving fuzzy systems from data streams in real-time". Proc. Int. Symp. On Evolving Fuzzy Systems (2006), pp. 26-32.

21. El-Koujok, M., Gouriveau, R. and Zerhouni, N. "Development of a prognostic tool to perform reliability analysis". Proc. ESREL - 17th SRA-Europe Conf., 2008.

22. Angelov, P. and Kasabov, N. "Evolving computational intelligence systems". Proc. 1st Int. Workshop on Genetic Fuzzy Systems, 2005.

23. Akaike, H., "A new look at the statistical model identification". IEEE Transaction Automatic Control, Vol. AC-19 (1974), pp. 716-723.

24. ftp://ftp.esat.kuleuven.ac.be/sista/data/process_industry (2009)

25. ftp://ftp.esat.kuleuven.ac.be/sista/data/mechanical (2009)

\section{Biography}

Mohamed El-Koujok received a MS in control systems in 2006 from the University of Paul Sabatier, Toulouse (France). Since october 2006, he is a $\mathrm{PhD}$ tudent at AS2M Department (Department of Automatic Control and Micro-mechatronic 
systems) of Femto-st Institute. His researcher concerns the developement of neuro-fuzzy based prognostics tools.

Dr. Rafael Gouriveau received his engineer degree from National Engineering School of Tarbes (ENIT) in 1999. He then got his MS (2000) and his Ph.D. in Industrial Systems in 2003, both from the Toulouse National Polytechnic Institute (INPT). During his $\mathrm{PhD}$, he worked in the field of risk management and dependability analysis. In September 2005, he joined the national high school of mechanics and microtechniques of Besançon (ENSMM) as Associate Professor. His main teaching activities are concerned with production, maintenance, manufacturing, and informatics domains. Nowadays, his research interests concern the development of industrial prognostics systems by using neurofuzzy methods, and the investigation of reliability modeling by using possibility theory.

Professor Noureddine ZERHOUNI received his engineer degree from National Engineers and Technicians School of Algiers (ENITA) in 1985. After a short period in industry as engineer, he received his Ph.D. Degree in Automatic Control from the Grenoble National Polytechnic Institute in 1991. In September 1991, he joined the National Engineering School of Belfort (ENIB) as Associate Professor. At this time, his main research activity was concerned with modelling, analysis and control of manufacturing systems. Since September 1999, Noureddine Zerhouni is Professor at the national high school of mechanics and microtechniques of Besançon. He founded and is responsible of the research group "Design and maintenance of mechatronic systems" (COSMI) of AS2M Department within Femto-st Institute. His main research activities are concerned with intelligent maintenance systems and emaintenance. Professor Noureddine Zerhouni has been and is involved in various European and National projects on intelligent maintenance systems like FP5 European Integrated Project of ITEA program (Information Technology for European Advancement) PROTEUS, NEMOSYS (Naval EMaintenance Oriented SYStem) with DCNS, and AMIMACFAME (Reliability Improvement of Embedded Machines) with ALSTOM and CEGELEC. 